\title{
GMR
}

\section{Association of glutathione S-transferase (GST) genetic polymorphisms with treatment outcome of cisplatin-based chemotherapy for advanced non-small cell lung cancer in a Chinese population}

\author{
H.L. Xiao' ${ }^{1}$ Z.T. Yang ${ }^{2}$, F. Han $^{3}$ and H.X. Wei ${ }^{1}$ \\ ${ }^{1}$ Department of Respiratory Medicine, \\ Zhengzhou Central Hospital Affiliated to Zhengzhou University, \\ Zhengzhou, China \\ ${ }^{2}$ The First Neurology Department, \\ the Affiliated Hospital of Hebei Engineering University, Handan, China \\ ${ }^{3}$ Department of Respiratory Medicine, \\ the First People's Hospital of Xinxiang, Xinxiang, China \\ Corresponding author: H.L. Xiao \\ E-mail: xiaohllh@126.com
}

Genet. Mol. Res. 15 (2): gmr. 15027320

Received September 14, 2015

Accepted December 7, 2015

Published April 27, 2016

DOI http://dx.doi.org/10.4238/gmr.15027320

\begin{abstract}
The aim of this study was to evaluate the association of GSTM1 null/present, GSTT1 null/present, and GSTP1 IIe105Val polymorphisms with the chemotherapy response and overall survival of advanced NSCLC. Two hundred and sixty-two patients with histologically confirmed advanced NSCLC (inoperable TNM stages IIIA, IIIB, and IV) were enrolled to this hospital-based study between May 2009 and May 2012. The GSTM1 null/present, GSTT1 null/present, and GSTP1 IIe105Val polymorphisms were genotyped by polymerase chain reaction coupled with restriction fragment length polymorphism. A logistic regression analysis revealed a correlation between the null genotype of GSTM1 and improved response to chemotherapy [odds
\end{abstract}


ratio $=1.82 ; 95 \%$ confidence interval $(\mathrm{CI})=1.06-3.14]$. Analyses with the Cox proportional hazards model also indicated that the null genotype of GSTM1 was associated with lower risk of death (hazard ratio $=0.40$; $95 \% \mathrm{CI}=0.23-0.69$ ). In conclusion, the null genotype of GSTM1 was found to be correlated with improved response to chemotherapy and lower risk of death in advanced NSCLC patients.

Key words: GSTM1; GSTT1; GSTP1; polymorphism; NSCLC; Chemotherapy

\section{INTRODUCTION}

Lung cancer is the most common type of cancer affecting men and women worldwide, with respect to both incidence and mortality; moreover, the incidence and mortality rates of lung cancer in women are ranked third and second overall (IARC, 2012). Approximately 1.8 million new cases of lung cancer have been reported in 2012 (12.9\% of the total), 58\% of which were reported from under-developed regions. Lung cancer patients showed the highest mortality rate among patients with malignant tumors in China in 2012; additionally, a large number of new patients with lung cancer $(652,842)$ have been diagnosed in China during 2012 (IARC, 2012). Non-small cell lung cancer (NSCLC) accounts for $80 \%$ of lung cancer. NSCLC patients display individualized clinical outcomes, despite presenting similar TNM stages (or being subjected to similar treatment methods), which suggests the possible role of genetic factors in determining the clinical outcome of NSCLC. An increasing number of studies have predicted the role of drug-metabolizing enzymes (DME) in affecting inter-individual variations in treatment reaction (Oguri et al., 2000; Joerger et al., 2012).

The human glutathione-S-transferases (GSTs) family belongs to the dimeric phase II metabolic enzymes, and it contributes to detoxify chemotherapeutic drugs or their metabolites (Oguri et al., 2000; Joerger et al., 2012). Allelic deletions in the GSTM1 and GSTT1 genotypes are associated with reduced enzyme activity (Strange et al., 2001). GSTP1 IIe105Val genetic polymorphism $\mathrm{h}$ is correlated with lower substrate-specific catalytic activity. Few previous studies have reported the association between polymorphisms in GST genes and prognosis of advanced NSCLC, and their results are inconclusive (Vlachogeorgos et al. 2008; Ada et al., 2010). In this study, we attempted to evaluate the association of GSTM1 null/present, GSTT1 null/present, and GSTP1 IIe105Val polymorphisms with the chemotherapy response and overall survival of advanced NSCLC.

\section{MATERIAL AND METHODS}

\section{Patients}

We recruited 262 patients with histologically confirmed advanced NSCLC from the Zhengzhou Central Hospital Affiliated to Zhengzhou University between May 2009 and May 2012. The diagnosis of NSCLC was confirmed in all patients by pathological examination. Patients with serious concomitant systemic disorders resulting in an inability to receive chemotherapy or with other serious infection and neutral system diseases that may affect the safety of patients were excluded from the study. 
All included advanced NSCLC patients received a cisplatin-based combination chemotherapy regimen. Chemotherapy was repeated every three weeks for six cycles at most. If a state of unacceptable toxicity or disease progression was reached, the chemotherapy treatment would be terminated. The treatment response using RECIST v.1.1 was used to evaluate the patients' chemotherapy efficacy when they completed at least two cycles of chemotherapy (Eisenhauer et al., 2009). Complete response (CR) or partial remission (PR) were considered as good response to chemotherapy, and stable disease (SD) or progressive disease (PD) were regarded as poor response to chmotherapy. The overall survival (OS) time was calculated from the date of chemotherapy to the date of death from any cause. All patients with NSCLC were followed up until May 2014. Signed written consent forms were obtained from all NSCLC patients and the control subjects prior to enrolment. The ethics committee of the Zhengzhou Central Hospital Affiliated to Zhengzhou University approved the performance of this study.

\section{DNA extraction and genotyping}

Peripheral blood samples $(5 \mathrm{~mL})$ were obtained from all NSCLC patients and control subjects; these were stored at $-20^{\circ} \mathrm{C}$ until further use. Genomic DNA was extracted using a standard blood mini kit (Qiagen, Venlo, Netherlands) according to the manufacturer protocols. The GSTM1 null/present, GSTT1 null/present, and GSTP1 IIe105Val genotypes were amplified by polymerase chain reaction (PCR) coupled with restriction fragment length polymorphism (RFLP). The primer sequences used in this reaction were designed using the Sequenom Assay Design 3.1 software, as follows: GSTM1 null/present, F-5'GGGTACTTGATTGATCTG-3' and R-5'-GCTTGTAGAAGCTCATGC-3'; GSTT1 null/ present, F-5'-CTTGGTACTTTCCACATCTC-3' and R-5'-CCGCATGATCAGCACATTA C-3'; and GSTP1 IIe105Val, F-5'-CCAGAAGAGAGGACAGCTCA-3' and R-5'-CAACT GCGTCCATCACC- 3 '. The reaction conditions were set as follows: initial denaturation at $94^{\circ} \mathrm{C}$ for $4 \mathrm{~min} ; 35$ cycles of denaturation at $94^{\circ} \mathrm{C}$ for $30 \mathrm{~s}$, annealing at $60^{\circ} \mathrm{C}$ for 30 $\mathrm{s}$, and extension at $72^{\circ} \mathrm{C}$ for $30 \mathrm{~s}$; and a final extension at $72^{\circ} \mathrm{C}$ for $7 \mathrm{~min}$. The restriction enzyme for GSTP1 IIe105Val was BsmAI. The digested fragments for GSTM1 present and GSTT1 present were 215 and $480 \mathrm{bp}$. The digested fragments were $176 \mathrm{bp}$ for GSTP1 IIe/IIe, were 176, 91 and 85 bp for GSTP1 IIe/Val, and were 91 and 85 bp for GSTP1 IIe/Val.

\section{Statistical analysis}

The baseline characteristics of the patients presented as numbers $(\mathrm{N})$ and percentages (\%).Logistic regression analysis was used to estimated the role of GSTM1 null/present, GSTT1 null/present, and GSTP1 IIe105Val polymorphisms in determining the response to chemotherapy was analyzed by; these results were expressed as odds ratios (ORs) and 95\% confidence intervals (CIs). Cox regression model was used to evaluate the correlation between GSTM1 null/present, GSTT1 null/present, and GSTP1 IIe105Val polymorphisms and overall survival of advanced NSCLC. The hazard ratio (HR) and corresponding $95 \%$ confidence interval $(95 \% \mathrm{CI})$ were calculated for each factor. Two-sided $\mathrm{P}$ values $<0.05$ indicated statistically significant differences. All statistical analyses were performed with SPSS statistical software (version 17.0, SPSS Inc. Chicago, IL, USA). 


\section{RESULTS}

The median age of patients with NSCLC was $58.42 \pm 8.50$ years. Eighty-five females (32.44\%) and $177(67.56 \%)$ males were included in our study. Among the 262 NSCLC patients, $168(64.12 \%)$ were smokers, $86(32.82 \%)$ were drinkers, and $43(16.41 \%)$ displayed a family history of cancer among the immediate relatives; approximately $40 \%(103,39.31 \%)$ of the included patients presented squamous carcinoma and $159(60.69 \%)$ were affected by adenocarcinoma. More than $50 \%$ of the patients $(143 ; 54.58 \%)$ showed good response to chemotherapy (Table 1).

\begin{tabular}{|c|c|c|}
\hline Variables & Patients & $\%$ \\
\hline \multicolumn{3}{|l|}{ Age (years) } \\
\hline$<55$ & 122 & 46.56 \\
\hline$\geq 55$ & 140 & 53.44 \\
\hline \multicolumn{3}{|l|}{ Gender } \\
\hline Female & 85 & 32.44 \\
\hline Male & 177 & 67.56 \\
\hline \multicolumn{3}{|l|}{ Tobacco smoking } \\
\hline Never & 94 & 35.88 \\
\hline Ever & 168 & 64.12 \\
\hline \multicolumn{3}{|l|}{ Alcohol drinking } \\
\hline Never & 176 & 67.18 \\
\hline Ever & 86 & 32.82 \\
\hline \multicolumn{3}{|c|}{ Family history of cancer in the first relatives } \\
\hline No & 219 & 83.59 \\
\hline Yes & 43 & 16.41 \\
\hline \multicolumn{3}{|l|}{ Histological types } \\
\hline Squamous Carcinoma & 103 & 39.31 \\
\hline Adenocarcinoma & 159 & 60.69 \\
\hline \multicolumn{3}{|c|}{ Response to chemotherapy } \\
\hline Good response & 143 & 54.58 \\
\hline Poor response & 119 & 45.42 \\
\hline
\end{tabular}

The association between GSTM1 null/present, GSTT1 null/present, and GSTP1 IIe105Val polymorphisms and response to chemotherapy in advanced NSCLC is shown in Table 2. The results of logistic regression analyses revealed that the null genotype of GSTM1 was correlated with improved response to chemotherapy (OR $=1.82 ; 95 \%$ CI: 1.06-3.14). However, the GSTT1, and GSTP1 IIe105Val polymorphisms were not associated with response to chemotherapy in advanced NSCLC.

By the end of the follow-up period, the null genotype of GSTM1 was associated with longer overall survival of NSCLC compared to the GSTM1-present genotype (18.55 months vs. 15.73 months) (Table 3). Analyses with the Cox proportional hazards model indicated that the null genotype of GSTM1 is correlated with a lower risk of death (HR $=0.40 ; 95 \% \mathrm{CI}$ : $0.23-0.69$ ). However, polymorphisms in the GSTT1 and GSTP1 genes did not contribute to the overall survival of advanced NSCLC.

\section{DISCUSSION}

Patients with advanced NSCLC present highly individualized responses to cisplatinbased chemotherapy. Previous studies have indicated that genetic factors may contribute to such response. Response to chemotherapy could be modulated by hereditary factors that 
Table 2. Association between polymorphisms in various glutathione S-transferase genes (GSTM1 null/present, GSTT1 null/present, and GSTP1 IIe105Val polymorphisms) and response to chemotherapy in advanced nonsmall cell lung cancer (NSCLC).

\begin{tabular}{|c|c|c|c|c|c|c|c|c|}
\hline SNPs & Patients & $\%$ & Good response & $\%$ & Poor response & $\%$ & OR $(95 \% \mathrm{CI})^{1}$ & P value \\
\hline \multicolumn{9}{|l|}{ GSTMI } \\
\hline Present & 163 & 62.21 & 80 & 55.94 & 83 & 69.75 & 1.0 (Ref.) & - \\
\hline Null & 99 & 37.79 & 63 & 44.06 & 36 & 30.25 & $1.82(1.06-3.14)$ & 0.02 \\
\hline \multicolumn{9}{|l|}{ GSTT1 } \\
\hline Present & 145 & 55.34 & 77 & 53.85 & 68 & 57.14 & 1.0 (Ref.) & - \\
\hline Null & 117 & 44.66 & 66 & 46.15 & 51 & 42.86 & $1.14(0.68-1.92)$ & 0.59 \\
\hline \multicolumn{9}{|l|}{ GSTP1 } \\
\hline $\mathrm{IIe} / \mathrm{IIe}$ & 118 & 45.04 & 62 & 43.36 & 56 & 47.06 & 1.0 (Ref.) & - \\
\hline $\mathrm{IIe} / \mathrm{Val}$ & 66 & 25.19 & 36 & 25.17 & 30 & 25.21 & $1.08(0.57-2.08)$ & 0.79 \\
\hline $\mathrm{Val} / \mathrm{Val}$ & 78 & 29.77 & 45 & 31.47 & 33 & 27.73 & $1.23(0.67-2.29)$ & 0.48 \\
\hline
\end{tabular}

${ }^{1}$ Adjusted for age, gender, tobacco smoking, alcohol drinking, family history of cancer among the immediate relatives, and histological type. $\mathrm{OR}=$ Odd's ratio; $\mathrm{CI}=$ confidence interval.

Table 3. Association between polymorphisms in various glutathione S-transferase genes (GSTM1 null/present, GSTT1 null/present, and GSTP1 IIe105Val polymorphisms) and overall survival of non-small cell lung cancer (NSCLC).

\begin{tabular}{|c|c|c|c|c|c|c|c|c|c|}
\hline Genotypes & Patients & $\%$ & Death & $\%$ & Alive & $\%$ & Median survival (months) & $\mathrm{HR}(95 \% \mathrm{CI})^{1}$ & P value \\
\hline \multicolumn{10}{|l|}{ GSTM1 } \\
\hline Present & 163 & 62.21 & 116 & 70.30 & 47 & 48.45 & 15.73 & 1.0 (Ref.) & - \\
\hline Null & 99 & 37.79 & 49 & 29.70 & 50 & 51.55 & 18.55 & $0.40(0.23-0.69)$ & $<0.001$ \\
\hline \multicolumn{10}{|l|}{ GSTT1 } \\
\hline Present & 145 & 55.34 & 94 & 56.97 & 51 & 52.58 & 16.76 & 1.0 (Ref.) & - \\
\hline Null & 117 & 44.66 & 71 & 43.03 & 46 & 47.42 & 17.29 & $0.84(0.49-1.43)$ & 0.49 \\
\hline \multicolumn{10}{|l|}{ GSTP1 } \\
\hline IIe/IIe & 118 & 45.04 & 79 & 47.88 & 39 & 40.21 & 16.62 & 1.0 (Ref.) & - \\
\hline IIe/Val & 66 & 25.19 & 44 & 26.67 & 22 & 22.68 & 16.91 & $0.99(0.50-1.98)$ & 0.97 \\
\hline $\mathrm{Val} / \mathrm{Val}$ & 78 & 29.77 & 42 & 25.45 & 36 & 37.11 & 17.32 & $0.58(0.31-1.08)$ & 0.06 \\
\hline
\end{tabular}

${ }^{1}$ Adjusted for age, gender, tobacco smoking, alcohol drinking, family history of cancer among the immediate relatives, and histological type. $\mathrm{HR}$, hazard ratio; CI, confidence interval.

lead to an increase in cell activity, which in turn results in biotransformation, accumulation of intracellular cisplatin, and weakened DNA repair. Enzymes belonging to the GST superfamily are phase II enzymes involved in the metabolism of a wide range of xenobiotics and drugs, including a variety of cytotoxic chemotherapeutic agents against cancer (Tew, 1994). In this study, we examined the association between GSTM1 null/present, GSTT1 null/present, and GSTP1 IIe105Val polymorphisms and response to chemotherapy, as well as the clinical outcome of advanced NSCLC. The results of our study indicated that the null genotype of GSTM1 was associated with improved response to chemotherapy and longer overall survival time compared to the GSTM1-present genotype.

The results of a study conducted by Tulsyan et al. (2013) indicated that the variant genotypes of GSTs could be associated with an improved response to chemotherapeutic drugs and a better overall survival time because of their reduced ability to detoxify the metabolites of drugs and carcinogens. Moreover, polymorphisms in the GST genes are believed to be associated with survival in colorectal cancer, breast cancer, and osteosarcoma (Kap et al., 2014; Liu et al., 2014; Goričar et al., 2015). Kap et al. (2014), in a study with 755 colorectal cancer patients, discovered that the presence of a homozygote of GSTM1 resulted in significantly poorer survival in the cancer patients, despite oxaliplatin treatment. On the other hand, GSTP1 polymorphisms were associated with the treatment outcome in 66 osteosarcoma patients (Goričar et al., 2015). 
Two studies have reported an association between polymorphisms in GST genes and the clinical outcome of NSCLC. Variants of GSTP1 are associated with improved survival in patients with advanced NSCLC (Ada et al., 2010). Vlachogeorgos et al. (2008) reported that GSTP1 expression could be a predictor of the survival of, and response to treatment in, 39 patients with advanced NSCLC. In this study, the null genotype of GSTM1 was found to be correlated with better response to chemotherapy and a lower risk of death in advanced NSCLC patients. These inconsistencies could be attributed to the various differences in ethnicities, source of patients, sample size, and chance.

The results of this study were subject to two limitations. Firstly, the hospital-based design of the study may have introduced a selection bias to our results. Secondly, the sample size included in this study was relatively small, which may limit the statistical power of our findings. Therefore, further studies with larger sample sizes must be performed in the future to validate our results.

In conclusion, the null genotype of GSTM1 was found to be correlated with better response to chemotherapy and a lower risk of death in advanced NSCLC patients. However, further studies with larger sample sizes are required to confirm the role of GST polymorphisms in the clinical outcome of NSCLC.

\section{Conflicts of interest}

The authors declare no conflict of interest.

\section{ACKNOWLEDGMENTS}

We thank the help from nurses in the Zhengzhou Central Hospital Affiliated to Zhengzhou University. The nurses help us to collect the medical record and blood samples from study subjects.

\section{REFERENCES}

Ada AO, C Kunak S, Hancer F, Bilgen S, et al. (2010). CYP and GST polymorphisms and survival in advanced non-small cell lung cancer patients. Neoplasma 57: 512-521. http://dx.doi.org/10.4149/neo_2010_06_512

Eisenhauer EA, Therasse P, Bogaerts J, Schwartz LH, et al. (2009). New response evaluation criteria in solid tumours: revised RECIST guideline (version 1.1). Eur. J. Cancer 45: 228-247. http://dx.doi.org/10.1016/j.ejca.2008.10.026

Goričar K, Kovač V, Jazbec J, Zakotnik B, et al. (2015). Genetic variability of DNA repair mechanisms and glutathioneS-transferase genes influences treatment outcome in osteosarcoma. Cancer Epidemiol. 39: 182-188. http://dx.doi. org/10.1016/j.canep.2014.12.009

International Agency for Research on Cancer (2012). http://globocan.iarc.fr/Pages/fact_sheets_population.aspx. Accessed Juanuary $1,2015$.

Joerger M, Burgers SA, Baas P, Smit EF, et al. (2012). Germline polymorphisms in patients with advanced nonsmall cell lung cancer receiving first-line platinum-gemcitabine chemotherapy: a prospective clinical study. Cancer 118: 24662475. http://dx.doi.org/10.1002/cncr.26562

Kap EJ, Richter S, Rudolph A, Jansen L, et al. (2014). Genetic variants in the glutathione S-transferase genes and survival in colorectal cancer patients after chemotherapy and differences according to treatment with oxaliplatin. Pharmacogenet. Genomics 24: 340-347. http://dx.doi.org/10.1097/FPC.0000000000000059

Liu J, Luo J, Wang Y, Li L, et al. (2014). Predictive potential role of glutathione S-transferases polymorphisms on prognosis of breast cancer. Int. J. Clin. Exp. Pathol. 7: 8935-8940.

Oguri T, Fujiwara Y, Katoh O, Daga H, et al. (2000). Glutathione S-transferase-pi gene expression and platinum drug exposure in human lung cancer. Cancer Lett. 156: 93-99. http://dx.doi.org/10.1016/S0304-3835(00)00447-X 
Strange RC, Spiteri MA, Ramachandran S and Fryer AA (2001). Glutathione-S-transferase family of enzymes. Mutat. Res. 482: 21-26. http://dx.doi.org/10.1016/S0027-5107(01)00206-8

Tew KD (1994). Glutathione-associated enzymes in anticancer drug resistance. Cancer Res. 54: 4313-4320.

Tulsyan S, Chaturvedi P, Agarwal G, Lal P, et al. (2013). Pharmacogenetic influence of GST polymorphisms on anthracycline-based chemotherapy responses and toxicity in breast cancer patients: a multi-analytical approach. Mol. Diagn. Ther. 17: 371-379. http://dx.doi.org/10.1007/s40291-013-0045-4

Vlachogeorgos GS, Manali ED, Blana E, Legaki S, et al. (2008). Placental isoform glutathione S-transferase and P-glycoprotein expression in advanced nonsmall cell lung cancer: association with response to treatment and survival. Cancer 114: 519-526. http://dx.doi.org/10.1002/cncr.23981 